\title{
Locoregional Breast Cancer Surgery: Is there a Role in the Presence of Metastatic Disease?
}

\author{
J Bisnath ${ }^{1 *}$, R Ramkissoon ${ }^{1}$ and J Ali, ${ }^{1,2}$ \\ ${ }^{1}$ Breast Surgery Unit, National Women's Health Clinic, St. James Medical Complex, Trinidad \\ ${ }^{2}$ Department of Surgery, St. Michael's Hospital, University of Toronto, Canada
}

*Corresponding author: Dr. Jacinta Bisnath, Breast Surgery Unit, National Women's Health Clinic, St. James Medical Complex, Trinidad, Tel: 8684729642, E-mail: jacintadbisnath@gmail.com

\begin{abstract}
Treatment of Stage Four Breast Cancer has traditionally excluded locoregional surgery, with the focus being on control of the distant disease. Much of what is applied in clinical practice is extrapolated from chemotherapy trials in the adjuvant setting, with taxanes and anthracyclines incorporated early on in the treatment course.

In unusual cases where there is response to chemotherapy, there exist no published consensus or guidelines. In the metastatic setting, available data show that the resection of the primary tumour decreases local progression and increases overall survival (OS) [1].

There is controversy regarding whether locoregional surgery has any role in the context of treatment of metastatic breast cancer. However, according to Rapiti, et al., surgery of the primary tumour (with negative surgical margins) in patients with metastatic breast cancer was significantly linked to more than fifty percent reduction in the breast cancer mortality, as compared to women who did not have surgical treatment [2].

We present a case of metastatic breast cancer, showing total response to chemotherapy, which was followed by locoregional surgery.
\end{abstract}

\section{Keywords}

Metastatic breast cancer, Locoregional surgery

\section{Abbreviations}

OS: Overall Survival; MMG: Mammogram; CTCAP: Computed Tomography of the Chest, Abdomen, Pelvis; PET: Positron Emission Tomography; CA15-3: Cancer Antigen 15-3; NCCN: National Comprehensive Cancer Centre Network; HR: Hormone Receptor; ER: Estrogen Receptor; PR: Progesterone Receptor; HER 2: Human Epidermal Growth Factor Receptor 2; PCR: Pathological Complete Response; EFS: Event Free Survival.

\section{Introduction}

There are no universally accepted treatment protocols for metastatic breast cancer, especially with regards to surgical intervention. Traditionally chemotherapy has been considered the mainstay. However, it is individually tailored to each patient, based on response and Eastern Cooperative Oncology Group (ECOG) Score. The use of locoregional surgery remains controversial with the efficacy of its role and the timing in patients, currently subject to ongoing investigations.

\section{Case Description}

Ms KM, a 47-year-old Afro-Trinidadian female was referred to the local National Radiotherapy Centre, with a diagnosis of Left Breast Cancer. Of significance, her mother was diagnosed with breast cancer at the age of 55 . She has no other relevant past medical, drug, allergy, social or gynaecological history.

The left breast lump was first noticed in May 2015, for which she consulted a private physician. On examination, a $1 \mathrm{~cm} \times 0.5 \mathrm{~cm}$, hard, mobile mass was palpated.

She was referred for a Mammogram (MMG) and Fine Needle Biopsy of the left breast mass.

\section{Investigations}

On MMG there was a $1.5 \mathrm{~cm}$ spiculated left breast mass. The Fine Needle Biopsy showed no evidence of malignancy.

An incisional biopsy was performed on $16^{\text {th }}$ December 2015, at the referring institution which showed,

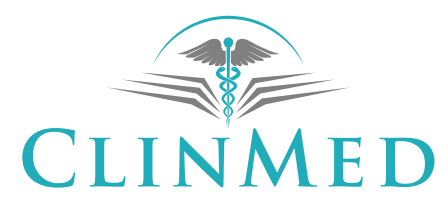

INTERNATIONAL LIBRARY
Citation: Bisnath J, Ramkissoon R, Ali J (2018) Locoregional Breast Cancer Surgery: Is there a Role in the Presence of Metastatic Disease?. Int J Oncol Res 1:005.

Accepted: June 09, 2018; Published: June 11, 2018

Copyright: (c) 2018 Bisnath J, et al. This is an open-access article distributed under the terms of the Creative Commons Attribution License, which permits unrestricted use, distribution, and reproduction in any medium, provided the original author and source are credited. 
Grade III Infiltrating Carcinoma of No Specific Type, multifocal areas suspicious but not definitive for lymphovascular invasion, tumour present at surgical margins; no ductal carcinoma in situ present, with a Nottingham Score of 8 . Immunohistochemistry revealed triple negative disease.

She was then referred to the National Women's Health Clinic - Breast Unit.

\section{Management course}

She presented on the $20^{\text {th }}$ January 2016 with visible scar on left breast. Nil other masses were palpated, and there was no appreciable axillary lymphadenopathy. She was booked for a Left Wide Local Excision + Axillary Lymph Node Clearance and for staging computed tomography of the chest, abdomen and pelvis (CT CAP) with contrast. CT CAP revealed metastatic disease as described below, and breast conservation surgery with axillary lymph node clearance was not done.

CT CAP done on $21^{\text {st }}$ January 2016 showed a six-millimetre subpleural nodule in the posterobasal right lower lobe, at least three one millimetre to three-millimetre nodules in the left lingual lobe, four-millimetre nodule in the anterior segment of the right upper lobe, two-millimetre nodule in the right middle lobe and one-millimetre nodules in the superior segment of the right lower lobes were suggestive of metastases. There was a focal hypoenhancing lesion $1.4 \mathrm{~cm} \times 1 \mathrm{~cm}$ on segment II of the liver suggestive of hepatic metastasis.

Following initial workup, chemotherapy with Adriamycin and Cyclophosphamide was planned for 4 cycles to be followed with Paclitaxel for 4 cycles. She went on to receive the four cycles of Adriamycin $60 \mathrm{mg} / \mathrm{m}^{2}$ and Cyclophosphamide $600 \mathrm{mg} / \mathrm{m}^{2}$ (each given in Day 1 of a 21-day cycle) between the $3^{\text {rd }}$ February 2016 and the $6^{\text {th }}$ April 2016. The only reported complication from her treatment was mild neutropenia which was treated with filgrastim injections.

Repeat CT CAP with contrast done on $16^{\text {th }}$ April 2016 showed a punctate one-millimetre pulmonary nodule in the anterior segment of the right upper lobe (decreased in size from four millimetres previously) in addition to a complete response of hepatic metastatic deposit, with -no other changes in interval study.

KM was then continued Paclitaxel chemotherapy $175 \mathrm{mg} / \mathrm{m}^{2}$ (given on day 1 of a 21-day cycle) between the $22^{\text {nd }}$ April 2016 and $29^{\text {th }}$ June 2016. There were no reported adverse events or toxicities.

Upon completion of this planned chemotherapy, a medical oncology consultation consensus lead to 3 further cycles of Paclitaxel being planned along with arrangement for a Positron Emission Tomography (PET) scan. The chemotherapy was received between $18^{\text {th }} \mathrm{Au}-$ gust 2016 and $29^{\text {th }}$ September 2016.

Positron Emission Tomography (PET) scan done on $17^{\text {th }}$ October 2016 showed -no evidence of recurrent or residual disease.

On assessment by the surgical team on $31^{\text {st }}$ October 2016, a repeat CT CAP was suggested in three to six months.

CT CAP repeated on $10^{\text {th }}$ April 2017 showed complete resolution of the previously mentioned right upper lobe pulmonary nodule with -no evidence of pulmonary, hepatic or bone metastases and no evidence of local disease recurrence.

A surgical consultation was held together with a multidisciplinary meeting and a decision to perform a left mastectomy with axillary lymph node clearance was made, which was done on $18^{\text {th }}$ July 2017.

Histological analysis of the excised breast revealed extensive hyalinized stroma, stromal edema, elastosis with presence of atrophic and scant glandular breast ducts and lobules, with no evidence of residual tumour or microcalcifications. Ten out of ten axillary lymph nodes were found to be benign. There was a total pathologic response.

Ms KM's case was discussed at Oncology Multidisciplinary Team meetings and close follow up has been planned in terms of management approach.

A third CT CAP done on $9^{\text {th }}$ October 2017, to monitor the metastatic lesions revealed:

Status post-mastectomy with no evidence of pulmonary or hepatic metastases. Throughout Ms KM's follow up, Tumour marker biochemistry (Ca15-3) has always been normal.

\section{Discussion}

Cancer is the second leading cause of death in the Caribbean, with breast cancer accounting for $14-30 \%$ of cancer deaths, making it the leading cause among women, two times higher than that compared to the United States of America [3].

Approximately $12 \%$ of breast cancer tumours are triple negative, are twice as common in African women and those with BRCA1 gene mutation and are associated with a poorer prognosis. Metastatic breast cancer has a reported five-year survival of $26 \%$ [4].

It is now widely accepted that the treatment of women with advanced metastatic breast cancer is non-surgical. Unfortunately, no randomized, controlled trials of chemotherapy versus best supportive care were conducted before chemotherapy for metastatic breast cancer was introduced. Such trials would now be considered unethical, as the response rate to first-line chemotherapy is so high (approximately 40\%) [5].

No single regimen has emerged as being the "gold standard" for the treatment of metastatic breast cancer. Anthracycline inclusion in Cooper type regimens have a 
slightly higher response rate and have been shown to reduce the hazard of dying and failure of treatment [6].

We do know, however, from published studies that cytotoxic-induced tumour shrinkage is associated in some women with symptom benefit and may prolong life [7].

According to National Comprehensive Cancer Network (NCCN) Guidelines 2016, chemotherapy regimens for metastatic breast cancer may include both single agent and combination regimens. The preferred single agents are anthracyclines, taxanes, antimetabolites or other microtubule inhibitors [8]. The combination agents include doxorubicin/cyclophosphamide, 4 cycles of which were administered to our patient coupled with 4 cycles of Paclitaxel, resulting in a complete radiological response.

There currently exists no specific guidelines for the surgical management of primary breast tumours in metastatic disease.

In a retrospective analysis using data from the NCCN, Dominici, et al. found that resection of the primary tumour did not confer any survival benefit in the Stage IV setting [9].

Furthermore, Badwe, et al., in a randomised study, suggests that there is also no benefit of locoregional surgery in terms of survival in patients with metastatic disease (except for bleeding/fungating tumours), even in those who have responded to front line chemotherapy, and this should not be considered a routine practice [10].

Due to the lack of clear cut guidelines, and our patient's complete radiological response, the decision was made by a multidisciplinary team to proceed with aggressive locoregional surgery in the form of mastectomy and axillary lymph node clearance.

This decision is supported by a retrospective study from Babiera, et al., which showed that surgical removal of the primary tumour resulted in a significant improvement in metastatic progression free survival as compared to those patients who did not have locoregional surgery [11].

Rapiti, et al., further reinforces this management choice in a study which showed that out of 300 patients, surgical removal of the primary tumour (with negative margins), in metastatic breast cancer, is linked to a > $50 \%$ reduction in breast cancer mortality [2].

San Gang-Wu, et al., (2017) in a retrospective study showed that the long-term survival benefit of surgery is greatest in those patients who are Hormone Receptor (HR)+/HeR2+ and HR+/HeR2-, with the lowest benefit to those patients who are triple negative. Regardless, overall survival benefit was still increased in triple negative patients receiving surgery as opposed to those who did not (24.7\% vs. $7.7 \%)$ [12].
The role of axillary lymph node surgery in these patients remains controversial, although, Taffurelli, et al., recommended that these patients undergo a sentinel lymph node biopsy intraoperatively, with axillary lymph node clearance being carried out during the same surgery for macrometastases (metastases $>2 \mathrm{~mm}$ in size) [13].

Postoperatively, the patient's histology from both the breast and axillary lymph nodes showed a complete pathological response to chemotherapy with no evidence of disease in both specimen.

There exist many definitions for pathological complete response (PCR), but complete eradication of cancer from the breast and axillary lymph nodes can be considered a surrogate end for patients with triple negative disease and is associated with improved event free survival (EFS) and overall survival (OS) [14].

Based on this definition, our patient achieved a pathological complete response after what is unconventional treatment. We are not able to state if this patient's outcome would be different if no locoregional surgery was performed. However, it seems prudent to offer this option to patients with the hope that a long term beneficial outcome will result.

\section{Conclusion}

Our case is unusual and demonstrates that our approach should be flexible in dealing with metastatic breast cancer. In the face of dramatic clinical response, consideration should be given to aggressive locoregional surgery, despite the presence of metastatic disease, as it may confer increased survival benefit to the patient.

The role of locoregional surgery in the presence of total disappearance of metastatic disease remains controversial. We have described our experience with this entity in our present manuscript but clearly large scale multi - institution trials are warranted to provide more specific and detailed guidance.

\section{References}

1. Kolben T, Kolben TM, Himsl I, Degenhardt T, Engel J, et al. (2016) Local Resection of Primary Tumor in Upfront Stage IV Breast Cancer 11.

2. Rapiti E, Verkooijen HM, Vlastos G, Fioretta G, Neyroud Caspar I, et al. (2006) Complete excision of primary breast tumor improves survival of patients with metastatic breast cancer at diagnosis. J Clin Oncol 24: 2743-2749.

3. Carpha, Taylor VC (2016) Breast cancer is two times higher in the caribbean compared to the USA CARPHA.

4. American Cancer Society (2015) Breast Cancer Facts \& Figures 2015-2016. American Cancer Society, Atlanta.

5. Jones A (1998) Non-Curative Chemotherapy for Breast Cancer. In: Slevin ML, Tate T, Cancer: How Worthwhile is Non-Curative Treatment? Focus on Cancer. Springer, London, 189-198.

6. Ahern R, Smith I, Ebbs S (1993) Chemotherapy and survival in advanced breast cancer: The inclusion of doxorubicin in Cooper type regimens. $\mathrm{Br} \mathrm{J}$ Cancer 67: 801-805. 
7. Ramirez A, Towlson K, Leaning M, Richards $M$, Rubens $R$ (1998) Do patients with advanced breast cancer benefit from chemotherapy? Br J Cancer 78: 1488-1494.

8. J Gradishar William, Anderson Benjamin, Balassanian Ron, L Blair Sarah, Burstein Harold, et al. (2015) Breast Cancer, Version 1.2016. J Natl Compr Canc Netw 13: 1475-1485.

9. Dominici L, Najita J, Hughes M, Niland J, Marcom P, et al. (2011) Surgery of the primary tumor does not improve survival in stage IV breast cancer. Breast Cancer Res Treat 129: $459-465$.

10. Badwe R, Hawaldar R, Nair N, Kaushik R, Parmar V, et al. (2015) Locoregional treatment versus no treatment of the primary tumour in metastatic breast cancer: An open-label randomised controlled trial. The Lancet Oncology 16: 13801388.
11. Babiera GV, Rao R, Feng L, Meric-Bernstam F, Kuerer HM, et al. (2006) Effect of Primary Tumor Extirpation in Breast Cancer Patients Who Present with Stage IV Disease and an Intact Primary Tumor. Ann Surg Oncol 13: 776-782.

12. Wu SG, Zhang WW, Sun JY, Li FY, Lin HX, et al. (2017) The survival benefits of local surgery in stage IV breast cancer are not affected by breast cancer subtypes: A population-based analysis. Oncotarget 8: 67851-67860.

13. Mario Taffurelli, Isacco Montroni, Donatella Santini, Monica Fiacchi, Simone Zanotti, et al. (2012) Effectiveness of sentinel lymph node intraoperative examination in 753 women with breast cancer: Are we overtreating patients? Ann Surg 255: $976-980$.

14. Cortazar $\mathrm{P}$, Zhang L, Untch $\mathrm{M}$, Mehta $\mathrm{K}$, Costantino JP, et al. (2014) Pathological complete response and long-term clinical benefit in breast cancer: The CTNeoBC pooled analysis. Lancet 384: 164-172. 